\title{
ENDERMOTERAPIA E ULTRASSOM TERAPÊUTICO ASSOCIADO À MASSAGEM MODELADORA NA REDUÇÃO DE MEDIDAS ABDOMINAIS
}

\author{
Pâmela Camila PEREIRA ${ }^{1}$ \\ Jennifer Luchesca Ferreira CÉZAR ${ }^{2}$ \\ Luís Henrique Sales OLIVEIRA ${ }^{3}$ \\ Marta Maria DELFINO ${ }^{4}$
}

\begin{abstract}
${ }^{1}$ Fisioterapeuta, Especialista em Fisioterapia Dermatofuncional-FEPI, Mestranda em Reabilitação do Equilíbrio Corporal e Inclusão Social -UNIAN. Docente do Curso de Fisioterapia do Centro Universitário de Itajubá-FEPI. pam_milaf@yahoo.com.br

${ }^{2}$ Fisioterapeuta, Especialista em Fisioterapia Dermatofuncional-FEPI. luchescaferreira@gmail.com

${ }^{3}$ Fisioterapeuta, Licenciado em Biologia, Mestre em Ciências Biológicas-UNIVAP e Doutor em Ciências - UNIFESP. Docente do Curso de Fisioterapia do Centro Universitário de Itajubá - FEPI. lhfisio@ig.com.br

${ }^{4}$ Fisioterapeuta, Especialista em Fisioterapia na Saúde da Mulher-UGF, Mestre em Ciências Biológicas-UNIVAP. Coordenadora do Curso de Pós-Graduação em Fisioterapia Dermatofuncional e Docente do Curso de Fisioterapia do Centro Universitário de Itajubá - FEPI. marta_delfino@ hotmail.com
\end{abstract}

\section{Recebido em: 25/04/2015 - Aprovado em: 15/08/2015 - Disponibilizado em: 30/10/2015}

\begin{abstract}
RESUMO
Introdução: A gordura localizada apresenta-se como um desenvolvimento irregular do tecido conjuntivo subcutâneo. Além dos meios convencionais como dieta hipocalórica e/ou exercícios físicos, muitos recursos podem ser utilizados para resolver esse distúrbio de insatisfação do corpo. O tratamento com Ultrassom associado à Endermoterapia possibilita efeitos de grande importância para redução de medidas. Objetivo: Avaliar os efeitos da massagem modeladora associada a recursos eletroterapêuticos na redução de medidas abdominais. Metodologia: A amostra incluiu 11 (onze) mulheres, com idade entre 20 a 35 anos, com adiposidade na região abdominal e que não estavam realizando nenhum tipo de tratamento estético e ou outras patologias associadas que interfiram no tratamento. Após a avaliação Fisioterapêutica Dermatofuncional, foram aplicadas 10 sessões do tratamento proposto por meio da aplicação da endermoterapia, ultrassom e massagens. Resultados: As pacientes apresentaram uma diminuição geral do perímetro corporal após as sessões de ultrassom $3 \mathrm{MHz}$, massagem modeladora associado à endermoterapia. Foi observada uma redução em todas as medidas propostas nas pacientes, com resultados significativos na perimetria, constatando assim que este número já é capaz de atingir objetivos na redução das medidas abdominais, mas ressalta que se o tratamento for continuado, os resultados serão otimizados e prolongados. Conclusão: Através da associação das técnicas, pôde-se verificar visível melhora do contorno corporal assim como melhora da textura da pele nos locais trabalhados à partir dos dados de redução de perimetria, a melhora do aspecto geral da pele através de registro fotográfico e a satisfação das pacientes.
\end{abstract}

Palavras chave: Lipodistrofia. Terapia por Ultrassom. Massagem. Modalidades de Fisioterapia. Gordura Abdominal

\begin{abstract}
Introduction: localized fat appears as an irregular development of the subcutaneous connective tissue. In addition to conventional means such as low-calorie diet and/or exercise, many resources can be used to resolve this body dissatisfaction disorder. Ultrasound treatment provides effects associated with Endermotherapy of great importance for mitigation. Objective: To evaluate the effects of normalizing massage associated with electrotherapeutic resources in reducing waist measurement. Methods: The sample included 11 (eleven) women, aged 20 to 35 years, with fat in the abdominal area and were not carrying any aesthetic treatment and or other associated diseases that interfere with treatment. After evaluating Physiotherapy Dermatofuncional were applied 10 sessions of treatment proposed by applying the Endermologie, ultrasound and massage. Results: The patients had an overall decrease in body circumference after the ultrasound sessions $3 \mathrm{MHz}$, shaping massage associated with Endermologie. A reduction was observed in all the action recommended in patients with significant results in perimetry, noting so this number is already
\end{abstract}


capable of reaching targets in reducing waist measurement, but notes that if treatment is continued, the results will be optimized and extended. Conclusion: Through the association of techniques, it was observed visible improvement in body contour and improves skin texture in places worked from perimetry data reduction, improvement in the overall appearance of the skin through photographic record and the satisfaction of patients.

Keywords: Lipodystrophy. Ultrasound therapy. Massage. Physical Therapy Modalities. Abdominal Fat.

\section{INTRODUÇÃO}

A valorização da imagem corporal ocupa cada vez mais um grande espaço dentre as preocupações da sociedade hoje, passando de uma preocupação relativa para uma preocupação evidente (BORGES, 2006; GUIRRO, GUIRRO, 2004).

$\mathrm{O}$ excesso de peso alcança valores relevantes e atinge todas as classes sócioeconômicas, estando diretamente relacionado com o índice de morbidade, incluindo hipertensão, diabetes mellitus tipo II e moléstias cardiovasculares. O aumento do percentual de gordura é consequência da elevação da ingestão calórica em detrimento da demanda energética (LEDUC, LEDUC, 2000).

Um dos principais alvos da Fisioterapia Dermatofuncional é o tecido adiposo, sendo uma forma especializada de tecido conjuntivo, formado por células chamadas adipócitos. A gordura localizada apresenta-se como um desenvolvimento irregular do tecido conjuntivo subcutâneo (CARDOSO, 2002).

A gordura adicional é armazenada nos lipócitos, que expandem no tamanho até que a gordura seja utilizada como fonte energética.
Quando são consumidas mais calorias, são produzidas as gorduras extras que estão armazenadas nos lipócitos (BORGES, 2006, MELLO et al., 2009).

Além dos meios convencionais como dieta hipocalórica e/ou exercícios físicos, muitos recursos podem ser utilizados para resolver esse distúrbio de insatisfação do corpo. O tratamento com Ultrassom (US) associado à Endermoterapia possibilita efeitos de grande importância para redução de medidas. A endermoterapia engloba equipamentos específicos baseados na sucção, age na recuperação da pele promovendo uma melhora na circulação, oxigenação, nutrição e eliminação de toxinas do tecido (GUIRRO, GUIRRO, 2004; HOPPE et al., 2010; TOGNI, 2006).

Milani (2006) afirma que a endermoterapia favorece a eliminação de toxinas, edemas e gorduras localizadas, por meio da sucção, mobilizando o tecido conjuntivo e tonificando as fibras elásticas.

O ultrassom de $3 \mathrm{Mhz}$ é definido por oscilações cinéticas ou mecânicas produzidas por um transdutor vibratório que quando aplicado sobre a pele, atravessa-a alcançando diferentes profundidades (AGNE, 2009; CHARTUNI, SOSSAI, TEIXEIRA, 2011). 
Segundo Sant'Ana (2010), o US tem como característica a geração de vibrações longitudinais, como consequência, um gradiente de pressão é desenvolvido nas células individuais obrigando os elementos da célula a se moverem. Ela afirma que o movimento gera uma micromassagem aumentando o metabolismo celular, o fluxo sanguíneo, o suprimento de oxigênio, facilitando o fluxo de nutrientes e a permeabilidade da membrana celular. (GONÇALVES et al., 2005).

Outra técnica coadjuvante na estética é a massagem modeladora, um procedimento que exerce efeito mecânico local decorrente da ação direta da pressão exercida no segmento massageado, e também ação reflexa indireta, por liberação local de substâncias vasoativas. Consiste em movimentos rítmicos, vigorosos e com maior pressão do que outras técnicas de massagem. Têm por objetivo aumentar a circulação sanguínea, favorecendo a demanda de catecolaminas responsáveis pela produção de substâncias que podem promover o esvaziamento das células adiposas. (GUIRRO, GUIRRO, 2004; TACANI, MACHADO, SOUZA, 2010).

A massagem desobstrui os poros, deixa a pele hidratada e mais delicada. Atua sobre as células mortas, apressando sua eliminação, estimula a circulação sanguínea ocorrendo hiperemia local. Atua no controle da retenção de líquido devido a sua atuação também no sistema linfático. No entanto não se encontra fundamentação científica para a massagem modeladora na atribuição do deslocamento de tecido gorduroso para determinadas regiões

(TACANI, MACHADO, SOUZA, 2010).

Para o sucesso dos tratamentos na fisioterapia dermatofuncional, é de extrema importância salientar a correta aplicação das técnicas, sendo imprescindível a presença do profissional qualificado e habilitado para a execução e compreensão dos procedimentos realizados (KEDE, SABATOVICH, 2004; MEDEIROS, 2004; MILANI, 2006).

\section{OBJETIVO}

Avaliar o efeito da endermoterapia e do ultrassom terapêutico associado da massagem modeladora na redução de medidas abdominais.

\section{CASUÍSTICA E MÉTODOS}

\subsection{Amostra}

A amostra incluiu 11 (onze) mulheres, com idade entre 20 a 35 anos, com adiposidade na região abdominal, e que não estavam realizando nenhum tipo de tratamento estético e/ou patologias associadas que interfiram no tratamento, tais como trombose venosa profunda, câncer, problemas renais ou circulatórios. Os critérios de exclusão foram à utilização de hormônios 
contraceptivos, a prática do tabagismo, etilismo e dieta alimentar irregular. $\mathrm{O}$ projeto de Pesquisa foi aprovado pelo Comitê de Ética do Centro Universitário de Itajubá sob Protocolo no 779.946/14.

\subsection{Instrumentos para Pesquisa}

A pesquisa foi realizada com o auxílio dos instrumentos listados abaixo, necessários aos procedimentos fisioterapêuticos:

-Equipamentos: Ultrassom 3MHz "Carci ${ }^{\circledR}$ ", Sonomed V;

-Aparelho Dermotonus Slim - Ibramed ${ }^{\circledR}$;

-Gel condutor para Ultrassom;

-Creme para Endermoterapia (Creme de Massagem Corporal Neutro sem Perfume D’água Natural ${ }^{\circledR}$ );

-Maca, Papel Toalha, Espátula, Fita métrica;

-Máquina fotográfica digital SONY - Cyber Shot $^{\circledR}$,

- Ficha de Avaliação Fisioterapêutica.

\subsection{Procedimentos da Pesquisa}

Foi realizada uma avaliação no início do estudo utilizando uma ficha de avaliação Dermatofuncional elaborada pelos pesquisadores. Foram analisados os dados de identificação, hábitos de vida, características físicas e perimetria da região abdominal e quadril. Os mesmos parâmetros descritos acima foram verificados após 10 sessões do protocolo de tratamento.
Após a avaliação, foi aplicado o tratamento proposto, por meio da aplicação da endermoterapia, ultrassom e massagens.

O Ultrassom foi usado com um gel condutor simples sem ativos, aparelho da marca $\mathrm{Carci}^{\circledR}$ e foram utilizados os seguintes parâmetros de modulação: frequência de $3 \mathrm{MHz}$ e intensidade de $1,6 \mathrm{~W} / \mathrm{cm}^{2}$, modo contínuo. Para o tempo de aplicação utilizouse uma regra na qual o tamanho da área a ser tratada foi dividido pelo tamanho da Area de Radiação Efetiva (ERA) do cabeçote. Desta forma, a região a ser tratada foi divida em 4 quadrantes, tempo de 20 minutos no total.

Para a Endermotonia, as participantes foram posicionadas em uma maca, em decúbito dorsal inicialmente e ventral posteriormente, com membro inferior semidespido usando apenas lingerie, e abdômen à mostra. Após, foi realizada a limpeza da região abdominal com álcool $70 \%$ e algodão. Para aplicação da técnica foi utilizado um creme para massagem D’água Natural ${ }^{\circledR}$ Neutro sem Perfume.

A sucção foi aplicada com intensidade variando de acordo com a tolerância de cada paciente (pressão disponível no aparelho que varia em torno de 0 a $-550 \mathrm{mmHg}$ ), com roletes grandes, com tempo de 10 (dez) minutos de aplicação no sentido favorável de retorno linfático. Conforme ilustrado na figura a seguir. 
Figura. 1 - Sentido da aplicação.

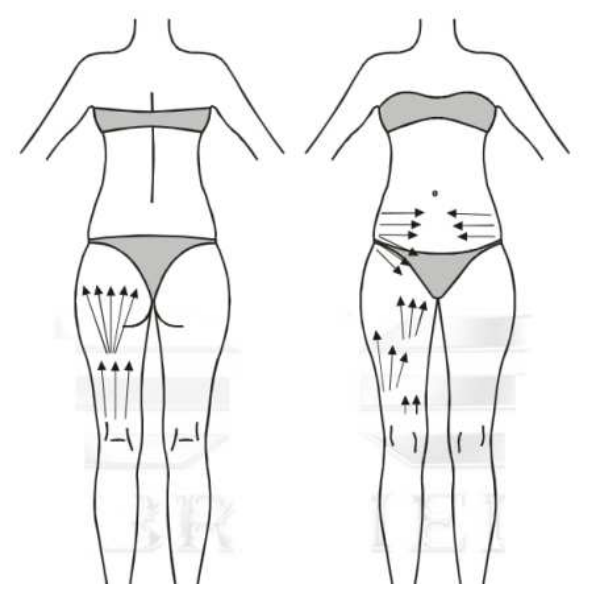

O protocolo foi aplicado em uma frequência de 1 (uma) vez por semana, durante 10 semanas, perfazendo um total de 10 (dez) atendimentos com duração de 60 minutos em média para cada sessão.

Após as 10 (dez) sessões, as pacientes foram reavaliadas pela ficha fisioterapêutica Dermatofuncional e realizada a coleta das medidas de circunferências conforme a proposta inicial.

A aferição das medidas de circunferência da cintura (CC), circunferência abdominal (CA), circunferência do quadril (CQ) foi realizada com fita métrica. Uma medida não antropométrica, mas de grande valia para o estudo foi à aferição da circunferência abdominal infra umbilical (CAIU), região localizada aproximadamente quatro centímetros abaixo da cicatriz umbilical que confere costumeiramente grande acúmulo de gorduras e fornece dados sobre a circunferência que abrange a região de flancos.

\subsection{Riscos e Benefícios}

A Endermoterapia não apresenta nenhum risco à saúde, apenas o cuidado de que alguns movimentos do aparelho podem romper alguns pequenos vasos. Suas contraindicações costumam ser as mesmas da massoterapia, isto é, hipertensão não controlada, tumores, hérnias, coágulos de sangue, uso de anticoagulantes, uso de cortisona injetável, diabetes, distúrbios renais, uso de marcapasso, reumatismo inflamatório, doenças vasculares e doenças infecciosas evolutivas. Como benefício, há a estimulação da circulação linfática e sanguínea, estimulação do processo imunitário, regeneração de tecidos, sensação de leveza e relaxamento além do combate ao Fibroedemagelóide e a flacidez.

\subsection{Análise dos Dados}

Ao término da coleta, os dados foram tabulados, analisados, discutidos e representados em gráficos.

Em seguida, o banco de dados foi exportado para o programa estatístico Bioestat 5.0 , onde os mesmos foram analisados com um nível de significância a $\mathrm{p} \leq 0,05$.

De acordo com Milani et al., (2006), a perimetria do membro tratado deve ser feita como uma rotina clínica, para a avaliação do resultado da terapia aplicada. 
Por fim, foram analisadas as imagens obtidas através de fotografias com base na literatura pesquisada.

\section{RESULTADOS E DISCUSSÃO}

Os resultados da perimetria estão detalhados no Gráfico 1, demonstrando à média por região, dos valores da mensuração na primeira avaliação, ou seja, antes do tratamento, e a média verificada depois do tratamento.

Gráfico 1 - Média por regiões da perimetria pré e pósintervenção fisioterapêutica.

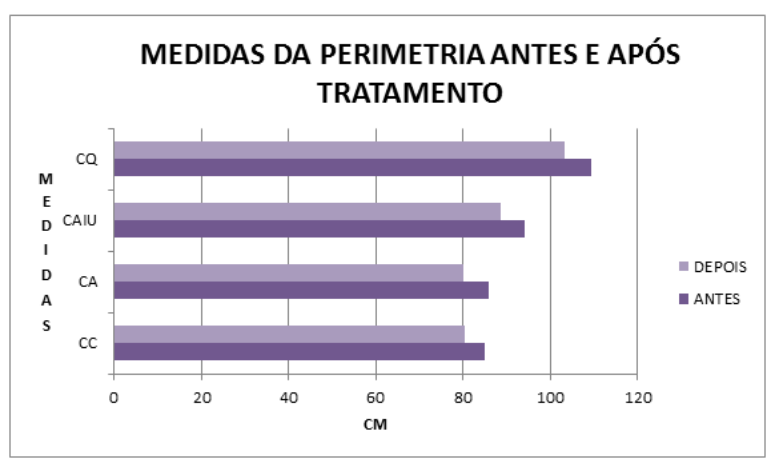

A partir da análise deste gráfico, podese perceber que as pacientes apresentaram uma diminuição geral do perímetro corporal após as sessões de endermoterapia, ultrassom $3 \mathrm{MHz}$ associado à massagem modeladora.

Chang et al., (1998) realizaram um estudo com 85 pacientes submetidas às sessões sobre a endermoterapia, após 7 sessões os pacientes já apresentaram reduções de medidas circunferenciais quanto de peso.

Já Tacani et al., (2010) utilizaram apenas a massagem na intervenção de 10 voluntárias, observaram redução significante da perimetria do quadril provavelmente pelo estímulo do metabolismo local.

Segundo Borges (2006) as técnicas usadas na massagem modeladora podem promover aumento da circulação sanguínea e linfática, aumento da nutrição do tecido, remoção de catabólicos, estímulo das funções viscerais, auxílio na penetração de ativos, mobilização e fluidificação da gordura, além de hiperemia cutânea local.

Não há referências na literatura pesquisada sobre as áreas que respondem melhor ou pior ao tratamento. Verificou-se, neste estudo, que a região do abdômen apresentou uma redução positiva com relação à perimetria aferida conforme os gráfico 2 . Sugere-se que isto tenha acontecido devido ao fato de ser a região que mais apresentava acúmulo de gordura localizada em todas as pacientes.

Gráfico 2 - Medidas de Circunferência Infra Abdominal

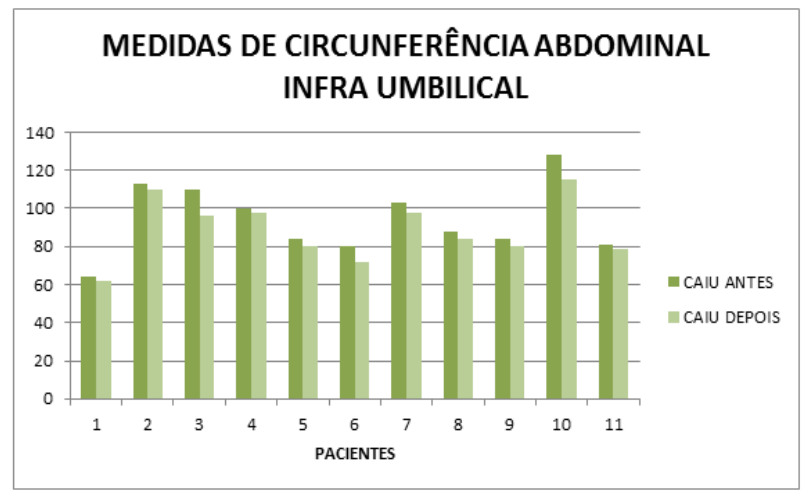

Com o intuito de observar visualmente a evolução do contorno corporal e o aspecto da pele antes e depois do tratamento, sob o ponto de vista qualitativo, a fotografia pode 
contribuir para a análise destes resultados. Como se pode observar nas figuras, as pacientes obtiveram uma ótima evolução decorrida da atuação conjunta dos equipamentos utilizados.

Fig. 2 - Registro fotográfico antes (esquerda) e depois (direita) da paciente após o tratamento. Vista Anterior.

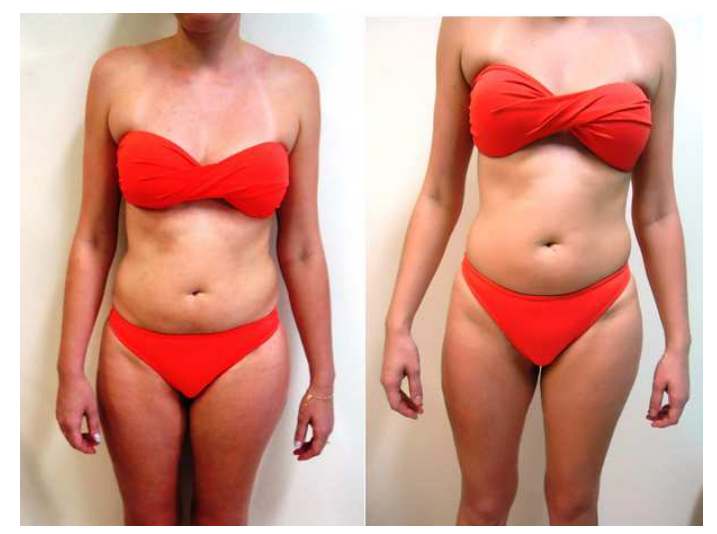

Fig. 3 - Registro fotográfico antes (esquerda) e depois (direita) da paciente após o tratamento. Vista Posterior.

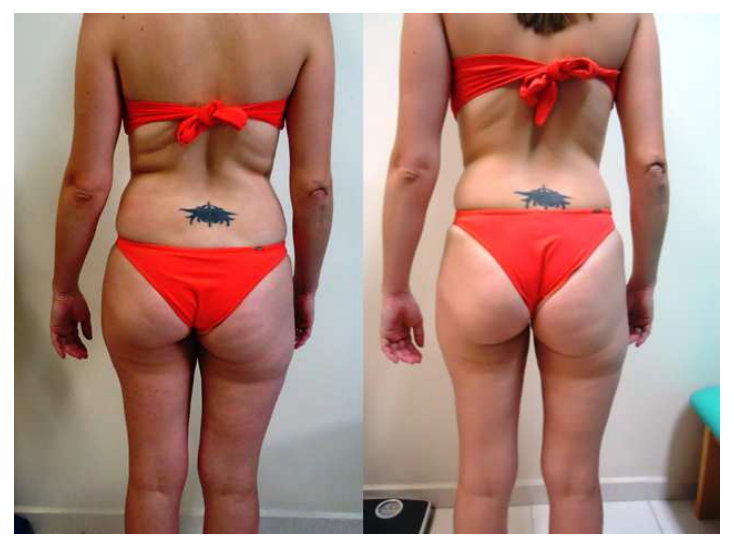

Pode-se observar na sequência de figuras: alteração do contorno corporal, melhora do aspecto da pele, irregularidades mais amenas, fibroses mais suaves ou quase invisíveis, assim como melhora geral da qualidade da pele, conforme Adcok et al., (1998) apud LUZ (2003) comprovam em estudo semelhante.
Nesse estudo, foi observada uma redução em todas as medidas propostas nas pacientes, com resultados positivos na perimetria, constatando assim que este número já é capaz de atingir objetivos na redução das medidas abdominais, mas ressalta que se o tratamento for continuado, os resultados serão otimizados e prolongados.

Milreau (2005) refere que a endermoterapia pode promover a eliminação de toxinas, aumento do aporte sangüíneo e oxigenação, melhor distribuição de gordura, modelamento da silhueta e, ainda, promover o relaxamento, entre outros efeitos. Lopes (2003) cita ações resultantes deste tratamento como a ação lipolítica (pela ação mecânica), a drenagem linfática e a eliminação de resíduos metabólicos (hipervascularização) associada ao desfibrosamento do tecido conjuntivo, garantindo uma melhor circulação dos fluidos), tonificação da pele (estímulo gerado no fibroblasto associado ao descongestionamento dos tecidos) e desestresse muscular (por diminuir tensões e otimizar as trocas tissulares).

Segundo Francischelli Neto, Francisquelli \& Junqueira (2001), não existe um peso ideal determinado para a estética feminina. O padrão de beleza depende do tempo e do local. A grande massificação das comunicações está levando à uma tendência padronizada da estética no mundo ocidental, relacionada a baixa gordura corporal e alta massa muscular. Os mesmos autores afirmam 
ainda que o "peso teórico ideal estético" deveria ser calculado para cada paciente, considerando as individualidades, a constituição física, a hereditariedade, a estética, a idade e um objetivo realista da capacidade de correção do excesso de gordura corporal e obtenção de massa muscular. Assim, novos estudos deveriam ser realizados para determinar índices de "peso ideal teórico estético", que pode ser diferente do peso teórico ideal e os tratamentos eficazes para cada caso.

É importante lembrar que, para um tratamento mais eficaz, que recupere a perfeita harmonia corporal, é imprescindível uma terapia combinada com diferentes recursos terapêuticos, exercícios físicos e reeducação de hábitos alimentares, não avaliados neste estudo, que objetivou somente analisar as respostas localizadas desencadeadas pelos recursos utilizados.

\section{CONCLUSÃO}

Por meio da associação das técnicas, pôde-se verificar visível melhora do contorno corporal assim como melhora da textura da pele nos locais trabalhados a partir dos dados de redução de perimetria, a melhora do aspecto geral da pele através de registro fotográfico e a satisfação das pacientes.

Sugere-se futuras pesquisas com uma amostragem maior para confirmação dos dados e com grupos controle, delimitados quanto á utilização isolada da massagem, da endermoterapia ou do ultrassom $3 \mathrm{MHz}$ para se ter um parâmetro de comparação mais fidedigno quanto à eficácia do plano de assistência individual de cada um destes.

\section{REFERÊNCIAS}

ADCOCK, D. et al. Analysis of the effects of deep mechanical massage in the porcine model. Plastic Reconstr Surg. Nashville, v.108, n.1, p. 233-240, July, 2001.

AGNE, J.E. Eu sei eletroterapia. Santa Maria: Palloti, 2009.

\section{BORGES, F.S. Dermato-funcional: modalidades terapêuticas nas disfunções} estéticas. São Paulo: Phorte, 2006. p- 541.

CARDOSO, E. A evolução e as novas técnicas utilizadas na estética corporal.

Revista Vida Estética, n.104. set, 2002.

CHANG, P. et al. Noinvasive mechanical body contouring: (endermologie) a oneyear clinical study update. Aesthetic Plast Surg. Austin, v.22, n.2, p. 145-153, Mar/Apr. 1998.

CHARTUNI, J.M.; SOSSAI, L.S.; TEIXEIRA, C.G. Efeitos do ultrassom 3MHz associado à ativos lipolíticos na adiposidade 
infra-abdominal: ensaio clínico randomizado.

Perspectivas online, 2011, 1: 79-12.

FRANCISCHELLI, M.; NETO, FRANCISCHELLI R.T.; JUNQUEIRA L.O.R. Hidrolipodistrofia: avaliação epidemiológica e uma proposta de classificação. Rev Med Estét Soc Bras Med Estét.2001;12:27-36.

GONÇALVES, W.L.S., CIRQUEIRA, J.P., SOARES, L.S, BISSOLI, N.S., MOYSES, M.R. Utilização da terapia ultra-sônica de baixa intensidade na redução da lipodistrofia ginecóide: uma terapia segura ou risco cardiovascular transitório? - Um estudo préclínico. An Bras Dermatol. 2005; 80(3): 352-359.

GUIRRO, E.; GUIRRO, R. Fisioterapia Dermato-Funcional. 3 ed. São Paulo: Malone; 2004.

HOPPE, S.; MARIN, M.F.; SIMÕES, N.P.; SIMIONATO, G. Fonoforese na Redução da adiposidade abdominal. Curitiba: Rev. Brasil Terapia e Saúde, 2010,1: 13-26.

KEDE, M.P.V. SABATOVICH, O. Dermatologia Estética. São Paulo: Atheneu, 2004.

LEDUC, A.; LEDUC, O. Drenagem Linfática. 2.ed. São Paulo: Manole: 2000.
LOPES, T.S. A utilização da endermologia no tratamento do fibro edema gelóide. Disponível em < http://www.fisioterapia.com/publicações/ultia ttrat.asp $>$ Acesso em 19 julho de 2014.

LUZ, C.M. Análise dos efeitos da terapia subdérmica não-invasica associada ao ultrasom de $3 \mathrm{MHz}$ no tratamento do fibro edema gelóide. Monografia de especialização. Florianópolis, 2003.

MEDEIROS, L.B. Abordagem Terapêutica. In: KADE, M.P.V.; SABATOVICH, O. Dermatologia Estética. São Paulo : Atheneu, 2004. 771p.

MELLO, P.B.; PICCININI, A.M.; BATISTA, E.; ROSA, L.H.; ROSA, P.V. Tratamento da gordura localizada através do uso de eletrolipólise e análise de ultra-sonografia. Nova Fisio, Rio de Janeiro, v.14, n.71, p.2023, dez. 2009.

MILANI, G.B.; JOÃO, S.M.A.; FARAH, E.A. Fundamentos da Fisioterapia dermatofuncional: revisão de literatura. Fisioterapia e Pesquisa, n.13, v.1, p.37-43, 2006.

MILREAU, P. Fisioterapia dermato funcional corporal. Paraná, 2005.

SANT'ANA, EMC. Fundamentação teórica para terapia combinada heccus ${ }^{\circledR}$ - Ultrassom 
e Corrente Aussie no tratamento da lipodistrofia ginóide e da gordura localizada.

Rev Bras Ciên Estét 2010; 1(1): 1-15.

TACANI, P.M.; MACHADO, A.F.P.; SOUZA, D.A. Efeito da massagem clássica estética em adiposidades localizadas: estudo piloto. Fisioterapia e Pesquisa.v.17, n.4, p. 352-357,2010.
TOGNI, A.B. Avaliação dos efeitos do ultrassom associado à fonoforese e endermologia no tratamento do fidro edema gelóide. Tubarão, 2006. Disponível em: http://www.fisiotb.unisul.br/Tccs/AlineBeatri zTogni/tcc.Acesso em: 20 de março de 2014. 